\title{
UN ESTUDIO SOBRE POLÍTICA PÚBLICA DEPORTIVA EN ARGENTINA: NOCIONES DEL DEPORTE SOCIAL EN EL MENEMISMO Y EL KIRCHNERISMO
}

\author{
UM ESTUDO SOBRE POLÍTICA PÚBLICA ESPORTIVA NA ARGENTINA: \\ NOÇÕES DE ESPORTE SOCIAL NO MENEMISMO E KIRCHNERISMO
}

\author{
A STUDY OF PUBLIC POLICY ON SPORTS IN ARGENTINA: NOTIONS OF \\ SOCIAL SPORTS DURING MENEMISM AND KIRCHNERISM
}

\section{Alejo Levoratti*}

\section{Palabras clave}

Deportes.

Políticas Públicas.

Factores Sociales.

Programas de gobierno.

\begin{abstract}
Resumen: En este trabajo se procura analizar las representaciones sobre el deporte social presentes en los lineamientos de la política deportiva desarrolla desde la "Secretaria de Deporte de la Nación" en dos gestiones específicas la de Fernando Galmarini, 1989-1992, durante la presidencia de Carlos Menem, y la de Claudio Morresi 2004-2014, durante las presidencias de Néstor Kirchner y Cristina Fernández de Kirchner. El trabajo privilegia el enfoque etnográfico al comprender las perspectivas situaciones de los actores. Se busca discutir teórica-metodológicamente con los trabajos locales inscriptos dentro de los estudios sociales sobre el deporte, donde este último término fue abordado como una arena social para el estudio de distintos tópicos, sin la producción de un análisis de esta categoría en términos nativos. Por ello, proponemos un análisis que contemple los sentidos asignados al "deporte social" en sus términos sociales, dando cuenta de los procesos de actualización y resignificación de esta categoría.
\end{abstract}

Resumo: Neste trabalho procuram-se analisar as representações sobre o esporte social presentes nas orientações da política esportiva desenvolvida pela Secretaria de Esporte da Nação em duas gestões específicas, a de Fernando Galmarini, 1989-1992, durante a presidência de Carlos Menem, e a de Claudio Morresi, 2004-2014, durante as presidências de Néstor Kirchner e Cristina Fernández de Kirchner. Privilegia-se o enfoque etnográfico ao compreender as perspectivas situacionais dos atores. Busca-se discutir teórico-metodologicamente com trabalhos locais inscritos dentro dos estudos sociais sobre esporte, onde esse último termo foi abordado como uma arena social para o estudo de distintos tópicos, não se produzindo uma análise dessa categoria em termos sociais. Por isto, propomos uma análise que contemple os sentidos assinalados ao "esporte social" em seus termos sociais, dando conta dos processos de atualização e ressignificação dessa categoria.

\begin{abstract}
This paper seeks to analyze the representation of social sport in the guidelines of public policy developed by Argentina's "Sport Department of the Nation" during two specific administrations: Fernando Galmarini's in 1989-1992, under the Carlos Menemadministration, and Claudio Morresi's in 2004-2014, during Néstor Kirchner's and Cristina Fernández de Kirchner's terms in office. The work uses the ethnographic approach to understand actors' perspectives. It aims to discuss - both in theory and methodologically - with local works under in the social studies about sports, where the latter term was approached as a social arena to study different topics, not producing an analysis of this category in native terms. Therefore, we propose an analysis that includes meanings assigned to "social sport" in its social terms, covering the process of renewal and reinterpretation of that category.
\end{abstract}

* Comisión de Investigaciones Científicas / Universidad Nacional de Quilmes. Universidad Nacional de La Plata. La Plata, Argentina. E-mail: levoratti@gmail.com

Recebido em: 04-03-2016 Aprovado em: 14-06-2016 (c) (1) (8) Licence 
[...] hablamos de deporte social para indicar que la gente participa de maneras diversas en el deporte, lo protagoniza, representa un espacio donde cada individuo se suma al "equipo"; indica una práctica en que jugar, cuidar el cuerpo y estar juntos constituye la meta. Es decir, con deporte social nos referimos a fenómenos generados desde la propia comunidad que expresan la necesidad de integración social, de pertenencia a un grupo, de reconocimiento de una identidad cultural. [...] (GALMARINI, 1992, p.82)

Estas conductas fijan la práctica deportiva como vehículo de integración social en la medida en que genera valores estimables de socialización y en que aparece como modo de relación entre personas y sectores sociales; genera modelos colaborativos, de competencia positiva y de mutuo conocimiento y ayuda a trasferir esos modelos a otras zonas de la vida en sociedad. Palabras de Fernando Galmarini, Secretario de Deportes de la Nación 1989-1992. (GALMARINI, 1992. p. 82).

El deporte social, centrado en la actividad física y la recreación, actúa como generador de situaciones de inclusión promoviendo la salud, la educación y la organización comunitaria de las personas sin discriminación de edad, sexo, condición física, social, cultural o étnica. Para ello, se busca extender la práctica deportiva a toda la población y formar recursos humanos con capacidad profesional y responsabilidad social. ${ }^{1}$

\section{INTRODUCCIÓN}

Entre los años 2008 y 2012 realicé trabajo de campo etnográfico en diferentes escuelas donde se desarrollaba un programa encuadrado dentro de la política socio-educativa de la Dirección General de Cultura y Educación de la provincia de Buenos Aires llamado "Patios Abiertos en las Escuelas". Alli indagué sobre las representaciones y prácticas de los profesores de educación física y funcionarios provinciales sobre el deporte. En ese contexto, aparecía de forma recurrente en los discursos de funcionarios y profesores la referencia a la categoría deporte social y la vinculación del deporte con la búsqueda de la inclusión. Ante dicha problemática comencé a indagar en los contextos que informaban este término para estos actores sociales, siendo significativos los lineamientos esbozados por la Secretaría de Deportes de la Nación. Al investigar en este organismo del Estado, en el período mencionado, encontré que la categoría deporte social oficia de ordenadora tanto material como simbólicamente de un segmento importante de la propuesta deportiva estatal, condensándose en este término, como veremos, un conjunto de sentidos presentados como unívocos y esencializados sobre lo que éste comprende, al mismo tiempo que en los relatos sobre estas prácticas eran incorporadas dentro de los lineamientos de la política nacional.

Con el objeto de analizar y desnaturalizar, para su mejor comprensión al deporte social desde la Secretaría de Deportes de la Nación, propongo realizar un trabajo comparativo sobre los alcances de este término en dos momentos particulares, donde el mismo oficia de organizador de la propuesta deportiva. La selección de estos dos periodos se fundamenta en que dentro de la narrativa de la categoría deporte social para los funcionarios que se desempeñaron entre 2008-2014 ésta forma parte de un proyecto políico que se inscribe en las antípodas del desarrollado durante la década de 1990 por el menemismo, considerado como neoliberal. Es por ello, que en este trabajo queremos comparar dichas gestiones, focalizando 
en la década de 1990 en la conducida por Fernando Galmarini, por ser la primera de este período neoliberal y porque es aquella en donde se identificaron la mayor cantidad de fuentes para ser trabajadas. El ejercicio de la comparación entre estos dos momentos nos posibilitará problematizar la modalidad de análisis y comprensión del deporte dentro de la política pública.

Al estudiar los sentidos del deporte en las políticas públicas seguimos el principio de Clifford Geertz (2003, p 33). cuando dice que: "Los Antropólogos no estudian aldeas (tribus, pueblos, vecindarios...); estudian en aldeas". En nuestro caso, la escala territorial delimitada por una determinada circunscripción estatal (La Nación- La Provincia- El Municipio) no se transforma en el objeto de estudio, sino que indagaremos en diferentes localizaciones institucionales procurando identificar los distintos componentes que informan las representaciones sobre el deporte social.

Por ello, partimos de la concepción de Ana Rosato y Fernando Balbi para el estudio de la política, quienes plantean que "[...] las representaciones sociales que en ellas se despliegan sólo pueden ser entendidas en función del análisis de procesos sociales y representaciones que, en principio, corresponderían a otros 'espacios"' (ROSATO; BALBI, 2003, p. 14). Teniendo en consideración el epígrafe, donde se presentan dos conceptualizaciones sobre el deporte social, expresadas por dos gestiones distintas de la Secretaría de Deportes de la Nación, queda al descubierto que las representaciones sobre estas prácticas no pueden ser analizadas solamente si tomamos en consideración la esfera "propiamente deportiva", política o de la gestión, sino que estas conceptualizaciones son resultantes de procesos sociales y se hallan informadas por diferentes espacios sociales. También, es importante considerar que tenemos en cuenta que las concepciones que presentamos deben ser circunscriptas en sus alcances a las fuentes trabajadas, a los actores involucrados y a los contextos indagados, no pudiéndose considerar que hacia dentro de cada una de las gestiones estudiadas se presente una uniformidad en las concepciones sobre el deporte social.

Asimismo, en este trabajo retomaremos el enunciado para el estudio del deporte expresado por Pierre Bourdieu (1990) y Roberto DaMatta (1982) quienes consideran que debe indagarse en los significados asignados por los sujetos sociales a esta categoría². Esta concepción es retomada también por Eduardo Archetti (1998) quien -recapitulando a Pierre Bourdieu en “¿Cómo se puede ser deportista?"- considera necesario el estudio de las apropiaciones sociales diferenciales de estas prácticas, permitiendo el estudio del deporte reflexionar sobre lo social y los mecanismos de creación de identidad. En estas producciones hallamos un enfoque teórico que comprende al fenómeno social deportivo como una arena social donde se pueden estudiar distintas problemáticas de las ciencias sociales. Esto se expresa también en la compilación realizada por Pablo Alabarces (2000, p. 11), quien en el estudio introductorio al libro "Peligro de Gol" -retomando a Jerome MacClancy (1996)- plantea que: "El deporte no es un 'reflejo' de alguna esencia postulada de la sociedad, sino una parte integral de la misma, más aún, una parte que puede ser usada como medio para reflexionar sobre la sociedad".

Por esto, este trabajo tiene como propósito indagar en las representaciones sobre el deporte social presentes en dos momentos de la política deportiva de la Secretaría de

2 Resulta importarte advertir que en este trabajo retomamos parcialmente la propuesta de los autores mencionados. De DaMatta nos diferenciamos sobre el hecho que éste considera al deporte como un reflejo de la sociedad, dado que supondría una coherencia que no es tal. De Bourdieu plantemos distancia sobre la asociación lineal establecida por el autor entre significados, funciones del deporte y posición en la estructura social. 
Deportes, prestando especial atención a los procesos de actualización y resignificación de esta categoría. Para ello en primer lugar, presentaremos sintéticamente la relación entre deporte y Estado en la Argentina, en segundo lugar nos concentraremos en analizar los significados en torno al deporte social en la gestión de la Secretaría de Deportes de la Nación a cargo de Fernando Galmarini entre 1989-1992, y en tercer lugar profundizaremos en esta categoría en este organismo entre 2008-2014. Para finalizar, reflexionaremos sobre los lineamientos compartidos y de distinción de cada una de las propuestas.

\section{EL DEPORTE EN LA ESFERA ESTATAL}

Este apartado tiene como propósito hacer una aproximación a la relación entre deporte y política estatal en el campo educativo y social. Al afrontar esta labor encontramos necesario ahondar en primera instancia en aquellas producciones que han indagado sobre este fenómeno social. En las producciones locales identificamos que sólo hacen mención a la problemática de forma tangencial, siendo por cierto un número reducido. Hasta el momento, en las Ciencias Sociales de Argentina no se ha producido un análisis sistemático de las políticas estatales del deporte. Por ello, las referencias que utilizaremos provienen de producciones que sólo tocan la temática de forma adyacente.

En este terreno, hallamos las contribuciones de Eduardo Archetti (2003, p. 42) que estudia la imagen nacional masculina considerando al fútbol y el polo como un diacrítico, dándole un rol protagonista a las prácticas del cuerpo en movimiento, considerando que el deporte se encuentra en un tipo de zonas libres de las tendencias ordenadoras de la sociedad por parte del Estado: "[...] la escuela, el servicio militar, el trabajo, las ceremonias públicas y rituales de nacionalidad". Asimismo, Archetti (2001, p.114) nos presenta el proceso de apropiación y de resignificación de los deportes en la construcción del imaginario nacional argentino. Por medio del estudio de distintas prácticas deportivas, como el automovilismo, el boxeo, el fútbol y el polo, nos muestra que "[...] si el deporte es pensado solamente a partir de la unanimidad no se puede entender la diversidad que éste produce [...] en la presentación de prácticas deportivas tan diferentes encontramos las bases de lo nacional [...]". Afirma que durante los diez años del peronismo "[...] no hubo, posteriormente, otros intentos sistemáticos de vincular el deporte con la nación a través de políticas estatales claras y articuladas" (ARCHETTI, 2003, p.116). Ambas producciones toman como referencia la obra de George Mosse, quien al analizar la construcción de las masculinidades durante el nazismo, sostiene que:

[...] la construcción de la masculinidad moderna depende, por lo tanto, de la relación entre "cuerpo y alma, de la moralidad y la estructura corporal" (MOSSE, 1996, p. 26). El esfuerzo físico y el cuidado corporal aparecen, de esa manera, no sólo como símbolos de la modernidad sino como algo que hay que cultivar y desarrollar, como una práctica individual y social que debe ser garantizada por el Estado y la sociedad civil. (MOSSE, 2001, p. 12).

Los trabajos que abordan la problemática del deporte (AISENSTEIN, 2006; ARCHETTI, 2001; FRYDENBERG, 2011) dan cuenta de la existencia de tensiones con otras prácticas corporales por su institucionalización en el sistema educativo. El deporte se deberá desarrollar en un principio, al margen de estas instituciones, en los clubes sociales y deportivos ${ }^{3}$. El Dr. Enrique Romero Brest instituyó el "Sistema Argentino de Educación Física" que consistía, en

3 En ese entonces el Ministerio de Educación dispuso que en las instituciones educativas se promoviera la creación de clubes atléticos dentro de cada establecimiento escolar (FRYDENBERG, 2011). 
sus principios, en ejercicios sin aparatos y juegos. Su introducción en el sistema educativo planteó permanentes tensiones - principalmente hasta la década del '30- con la educación física de tradición militarista que proponían principalmente los Maestros de Gimnasia y Esgrima del Ejército (SCHARAGRODSKY, 2011). A partir de este momento, se produjo un giro hacia los deportes como contenido central de la disciplina, orientación que se mantiene hasta la actualidad. Esta última tendencia se consolida a partir de la década del cuarenta debido a diversos factores tales como las críticas al Sistema Argentino de Educación Física, a la formación que imparte el Instituto Nacional de Educación Física y a la figura de Romero Brest; la creación de la Dirección Nacional de Educación Física a cargo de un ex deportista como César Vázquez; la popularidad del deporte fuera de la escuela y a profesores deportivistas que actuaban como docentes (AISENSTEIN, 1998; SCHARAGRODSKY, 2004). En este período se le suman una serie de beneficios a la educación de los cuerpos con los cuales son asociados los deportes, entre los que se pueden destacar: la libertad de movimientos, la iniciativa individual que da lugar cada situación de juego y el placer que emana de lo lúdico, que responde a las expectativas de nuevos sectores sociales que desean una formación para sus hijos como individuos emprendedores, reconociendo esos beneficios en los sports (AISENSTEIN, 2006).

Analizando las leyes educativas que marcaron el rumbo de la política educativa nacional y de la provincia de Buenos Aires durante las últimas dos décadas encontramos que en la Ley Federal de Educación, sancionada en el año 1993, se hace mención para los tres primeros niveles educativos (Inicial, Educación General Básica y Polimodal) la incorporación de la educación física y el deporte para el desarrollo integral de la persona y la preservación de la salud psicofísica, estando incorporadas las prácticas deportivas desde el nivel inicial, como primer estamento para su fomento. Con la sanción en el año 2006 de la Ley de Educación Nacional №26.206 desaparece la referencia hacia las prácticas deportivas, manteniéndose presente la educación física. La educación física procura alcanzar el objetivo (en los niveles Inicial, Primario y Secundario) de ofrecer una formación corporal y motriz contribuyendo para el desarrollo armónico de todos/as los/as niños/as e integral de los adolescentes. Para el nivel Inicial y Primario se destaca el rol del juego "como actividad ineludible para el desarrollo cognitivo, afectivo, ético, estético, corporal y social" (Ley 26.206, Artículos 26 y 27 Inciso c). En la educación secundaria (ex polimodal) se mantiene la incorporación y referencia a la educación física, a las prácticas deportivas y a la recreación al momento de hacer remisión a las propuestas "extracurriculares" con el propósito de lograr la "inclusión de adolescentes y jóvenes no escolarizados en espacios escolares no formales como tránsito hacia procesos de reinserción escolar plena" (artículo 32 Inciso e y f). En estos últimos cambios de legislación a nivel nacional se advierte un nuevo corrimiento en la incorporación de las prácticas deportivas, orientándose preponderantemente hacia programas, por fuera de las propuestas curriculares, que buscan la inclusión de adolescentes y jóvenes.

El desarrollo del deporte en el ámbito estatal no se circunscribe a su incorporación al sistema educativo, dado que estas prácticas se encontraron en distintos momentos históricos asociadas con diversas carteras ministeriales. Hallamos vínculos con el Ministerio de Bienestar Social/Acción Social, organismo del cual depende la Secretaría de Deportes de la Nación hasta 1989 cuando es transferida a la órbita de la Presidencia de la Nación. Al final del gobierno del presidente Fernando De la Rúa, 2001, se crea el Ministerio de Turismo, Cultura y Deporte, el cual tendrá una duración de tres meses. A partir del 2002 la Secretaría de Deportes vuelve a depender de la Presidencia de la Nación, siendo traspasada en diciembre del año 2007 al 
Ministerio de Desarrollo Social de La Nación, al momento de asumir la presidencia de la nación Cristina Fernández de Kirchner.

En las distintas propuestas analizadas, la Ley 20.655 de "Promoción de las actividades deportivas en todo el país", sancionada en marzo de 1974, será -como veremos- una referencia permanente de las fundamentaciones de las distintas políticas deportivas nacionales. Esta normativa define como principios generales el deporte:

\footnotetext{
[...] como factor educativo coadyuvante a la formación integral del hombre y como recurso para la recreación y esparcimiento de la población [...] como factor de la salud física y moral de la población [...]. Promoción de una conciencia nacional de los valores de la educación física y del deporte y la implementación de las condiciones que permitan el acceso a la práctica de los deportes de todos los habitantes del país [...]. (ARGENTINA, 1974, p.1)
}

Esta ley, que pregona una concepción esencialista sobre el deporte, carga a este concepto de una serie de valores vinculados con la formación del "hombre", principalmente, en términos físicos y morales. También es importante destacar su utilización como un "factor de la salud física y moral de la población".

La reglamentación de esta Ley fue iniciada en el año 1989 con el decreto presidencial №1.237 que regula la creación del Consejo Nacional del Deporte, las regiones deportivas, el consejo de regiones y el consejo de coordinación, dotando de las competencias en lo relativo a la orientación, fomento, promoción, fiscalización, asistencia y seguridad de las actividades deportivas a la Secretaría de Deportes. Asimismo resulta importante traer a la discusión estos lineamientos porque en ellos encontramos categorías que - como veremos - transcienden las gestiones, observándose continuidades y rupturas en las mismas.

Como vemos la inscripción del deporte en la esfera estatal se efectuó en diferentes agencias y con lineamientos particulares en cada uno de los casos, identificándose concepciones particulares en casa uno de los organismos estudiados.

A continuación desarrollaremos las representaciones sobre el deporte social presentes en dos momentos históricos particulares de la Secretaria de Deportes de la Nación.

\section{EL DEPORTE SOCIAL PARA LA SECRETARÍA DE DEPORTES DE LA NACIÓN ENTRE 1989-1992}

La presidencia de Carlos Saúl Menem (1989-1999) se caracterizó por la realización de reformas de carácter político, económicas, sociales de corte neoliberales. Para los funcionarios que acompañaron a Carlos Menem, el Estado era "ineficiente" porque carecía de una lógica mercantil. Al mismo tiempo se produjo un proceso de "descentralización" y la privatización de las empresas estatales y de servicios públicos. Estas transferencias tenían como argumento la reducción de la burocracia estatal, lo cual era una estrategia para volver más "eficiente" al Estado y la reducción del gasto público. En materia social, la acción del Estado se limitaba a la ejecución de programas sociales hacia determinadas poblaciones focalizadas, rompiéndose el criterio universalista anterior, lo que "[...] era un instrumento efectivo a la hora de neutralizar los hábitos no modernos" (FREDERIC, 2004,p. 78). Ingresando en el ámbito deportivo advertimos que se desempeñaron tres secretarios de deportes de la Nación: el primero fue Fernando "Pato" Galmarini entre 1989-1992, continuó Livio Forneris (1992-1995) y, entre 1996 y 1999, 
se desempeñó el ex. Puma ${ }^{4}$ Hugo Porta 5 . Para la era Galmarini, las prácticas deportivas se organizaron en dos grande grupos Deporte de alto rendimiento y Deporte Social ${ }^{6}$. El entonces secretario escribió un libro llamado "Deporte, política y cambio" (GALMARINI, 1992) donde relata los lineamientos y logros de su gestión. El autor construye una narrativa sobre el deporte en la Argentina y los lineamientos de las políticas deportivas que llevaron a cabo durante los primeros años de la década del '90. La edición del libro fue prologada por el entonces presidente Carlos Saúl Menem, quien destacaba que:

[...] El deporte es una celebración constante. Una conjura de alegría popular, fervor y fiesta. El deporte, además, nos hace mejores y nos enseña a vivir.

Porque el deporte no es solamente una fuente de regocijo: es también una enseñanza de vida, un modelo. El deporte enseña a trabajar con modestia y en soledad, sin esperar nada de nadie y el tácito mandato de exigirse todo a sí mismo. El deporte enseña a ponerse cada vez metas más altas. A no darse jamás por vencido. El deporte enseña a tolerar la derrota, a ponerse de pie y seguir adelante. Enseña también a tolerar el triunfo y ser generosos. El deporte enseña a amar una bandera, a jugarse por entero. El deporte enseña con las dificultades. A convivir con los otros. A ser pacientes. A perseverar. El deporte enseña a insistir cuando todo parece perdido [...].

Por ese camino, el camino del esfuerzo, de la disciplina y de la sana alegría, el deportista purifica su cuerpo y enaltece su alma ...] Firma Carlos S. Menem. (GALMARINI, 1992, p. 14)

Esta continuidad planteada en la concepción del deporte, donde Galmarini se referencia de forma permanente en la Ley del Deporte № 20.655, representa en su perspectiva las ideas que Perón tenía sobre esta práctica, colocando en forma reiterada la siguiente cita adjudicada al ex mandatario:

Nosotros, desde el gobierno, pensamos en la necesidad de expandir extraordinariamente el deporte, porque éste es el forjador de un cuerpo sano y un espíritu virtuoso.

También pensamos que, inculcar desde la infancia y la juventud la práctica del deporte es, quizás, la mejor y única escuela para la formación de hombres. Eso es lo que el país necesita. Hombres fuertes y virtuosos para que puedan servir a la comunidad en la forma más completa y más perfecta. (GALMARINI,1992, p. 39)

Ampliando en el desarrollo sobre este término, Galmarini (1992, p. 59) destacaba que:

[...] la sociedad genera siempre anticuerpos para combatir sus males. El deporte puede funcionar como uno de esos anticuerpos en tanto ofrece un espacio para encontrarse con los otros al calor de una afición compartida que, en ocasiones, se transforma en el lazo más duradero de la amistad; reúne al padre y al hijo en torno a un lenguaje común que puede saltar brechas generacionales; entrega al joven un modelo de adulto en el que identifica el valor, el esfuerzo, la victoria honesta y la dimensión lúdica [...].

\footnotetext{
4 Los pumas es el nombre con el cual se conoce al equipo nacional de Rugby del cual Porta fue jugador y capitán, asimismo se formó como arquitecto y se desempeñó como embajador en Sudáfrica entre 1991-1996.

5 Recordemos como planteamos más arriba que en este momento la Secretaría de Deportes dependía directamente de la Presidencia de la Nación.

6 Galmarini fue miembro de distintas agrupaciones políticas peronistas en la década de 1970 como: Descamisados, Montoneros, Lealtad; luego ejerció el cargo de Diputado nacional por el Frente Justicialista Federal en el período comprendido entre 1995-1999, presidiendo la comisión de deportes de dicha cámara.
} 
En los capítulos sucesivos, Galmarini se concentró en desarrollar los lineamientos de su gestión, manifestando en forma reiterada la ineficiencia que caracterizó al Estado en la administración de los recursos económicos en materia deportiva, haciendo énfasis en la discrecionalidad y en las prácticas clientelares que habrían realizado tanto civiles como militares en la distribución de los fondos. En ese sentido, considerando el análisis de Sabina Frederic (2004) quien analiza la incursión del modelo neoliberal en la Argentina a partir de la crisis económica de 1989, dejando al descubierto como éste es adaptado a las racionalidades y problemas locales. La autora muestra como Carlos Saúl Menem justifica su implementación en términos morales. En este sentido, al examinar la retórica de Menem sobre las modificaciones del Estado en materia económica y política, ellas se fundamentan por atacar la ineficiencia en la administración propiciando la figura del experto en su gestión. En este proceso, el papel que había desempeñado el Estado como empresario administrando los servicios públicos, resultaba incompatible para los objetivos neoliberales (FREDERIC, 2004, p.77). Para esos funcionarios, el Estado era ineficiente porque carecía de una lógica mercantil. Estas transferencias tenían como argumento la reducción de la burocracia estatal, lo cual era una estrategia para volver más "eficiente" al Estado y reducir el gasto público, como planteamos más arriba. Volviendo al terreno deportivo, ello se expresa en los principios de su propuesta donde el Estado no es el único responsable por el financiamiento del deporte nacional, por eso plantea como necesaria la participación del sector empresarial y de la sociedad civil, empleando para ello una retórica similar a la del presidente. Además en este período la inscripción de la temática deportiva dentro del Estado nacional modificará su estatus, perdiendo su rango de secretaria por dos años, como consecuencia de los distintos cambios en la estructura orgánica de la administración pública direccionados hacia la reducción de la cantidad de dependencias, con el argumento de reducir el gasto. La propuesta política ligada al deporte se centra, por un lado, en una dimensión llamada deporte social y, por el otro, en una definida como deporte de alto rendimiento. Galmarini (1992, p. 82) destacó que:

[...] hablamos de deporte social para indicar que la gente participa de maneras diversas en el deporte, lo protagoniza, representa un espacio donde cada individuo se suma al "equipo"; indica una práctica en que jugar, cuidar el cuerpo y estar juntos constituye la meta. Es decir, con deporte social nos referimos a fenómenos generados desde la propia comunidad que expresan la necesidad de integración social, de pertenencia a un grupo, de reconocimiento de una identidad cultural [...].

Esta categoría de deporte social se construye y es referenciada de forma directa por Galmarini a los propósitos sociales incorporados durante el Peronismo, en el cual se "[...] organizan los torneos intercolegiales, se promociona el deporte universitario, militar, el sindical. $Y$ es al deporte social a quien le correspondía la misión fundamental de integrarse con salud y educación creándose, para ello, los campeonatos Evita" (GALMARINI, 1992, p.43).

El deporte social para Galmarini concentra las diversas prácticas masivas del cuerpo que realiza la sociedad por fuera del deporte de alto rendimiento. Estas prácticas se desarrollan en tres formas: el deporte formal es aquel que se efectúa en los clubes que tienen en cuenta la dimensión competitiva y bajo la regulación de una federación deportiva 7 . En segundo lugar está el modelo del gimnasio, donde "[...] no se privilegia la dimensión competitiva, sino la práctica misma", enumerando una serie de prácticas del cuerpo tales como "[...] diversos tipos de gimnasia, tenis, paddle, artes marciales". En tercer lugar, el modelo del gimnasta solitario o del 
pequeño grupo sin pertenencia; este último se diferencia del gimnasio por no tener tecnología y no existir contrato (es el caso de las personas que salen a correr o aquellos pequeños grupos que se reúnen a jugar el fútbol) (GALMARINI, 1992, p. 83-84).

Del desarrollo de los modelos encontramos, por un lado, que al hacer referencia al deporte se lo asocia con una diversidad de prácticas de disímil procedencia. Esta categoría es usada para indicar a prácticas culturales del cuerpo en movimiento que se la coligarán con una serie de valores que son asignados como intrínsecos como la "[...] solidaridad, diversificación de los vínculos sociales, espíritu de grupo, la valoración del esfuerzo y la constancia" (GALMARINI, 1992, p. 82). Para presentar estos atributos se hace permanente mención al deportista de alto rendimiento como su máximo exponente, pero es propio aclarar que no se presenta una relación de continuidad en las trayectorias desde el deporte social al alto rendimiento. La importancia de la realización de estas prácticas, de deporte social, se encuentra fundamentada en el proceso de transferencia de dichos valores como expresara Galmarini (1992, p. 83) "[...] generar modelos colaborativos, de competencia positiva y de mutuo conocimiento y ayuda a transferir esos modelos a otras zonas de la vida en sociedad".

Al mismo tiempo se sientan las bases para diferenciarla de la dimensión del alto rendimiento, en donde uno de los puntos de divergencia sustancial es el nivel de las exigencias competitivas y la dedicación del deportista. Dado que en el deporte social la competencia entre deportistas puede tener lugar, de acuerdo al ámbito de desarrollo, estas prácticas se encontrarían en un nivel previo/inferior o por fuera de las exigencias del deportista de alto rendimiento.

Siguiendo a Galmarini (1992), dentro del deporte social se concentran las siguientes áreas: en primer lugar, la "iniciación deportiva", en cuyo desarrollo asigna un rol protagónico a la educación física a partir de la escolarización de estas prácticas y a la realización de torneos intercolegiales. La segunda es "el deporte y el desarrollo económico", que incorpora al deporte como espectáculo. La tercera es aquella que vincula al "deporte y las relaciones internacionales", área que le asigna a esta práctica una suerte de "diplomacia informal". En cuarto lugar, presenta el área de "deporte y salud"; y por último, el "espectáculo deportivo", aquel que se encarga de la "prevención y la seguridad".

Como se puedo observar en el apartado, durante la gestión de Galmarini el deporte social agrupó las actividades corporales que se desarrollaban por fuera del alto rendimiento, recurriendo regularmente a las palabras o ideas que expresaba Juan Domingo Perón para significar y legitimar estas prácticas. Asimismo a la hora de fundamentar su propuesta política deportiva se hace uso de una determinada retorica asociada al neoliberalismo, focalizando los argumentos en la ineficiencia del Estado en la administración, habilitando ello la participación de distintos sectores económicos y la tercerización de ella.

\section{EL DEPORTE SOCIAL PARA LA SECRETARÍA DE DEPORTES DE LA NACIÓN ENTRE 2007-2014}

El 25 de mayo de 2003 asume Néstor Kirchner como presidente de la Nación, su mandato se caracterizó por la renegociación de la deuda externa, impulsar el comercio interior, políticas sobre los derechos humanos, fuerte inversión en materia educativa y la construcción de lazos con los países de la región. Asimismo se inician diversos programas con el objeto 
de ampliar distintos derechos sociales, que serán continuados y profundizados por Cristina Fernández de Kirchner quien asumirá la máxima magistratura en diciembre de 2007. En materia de política social la problemática de la inclusión es colocada en el foco de la acción, creándose y reorientándose distintos programas hacia tal logro. A partir de su asunción la Secretaría de Deportes es transferida a la órbita del Ministerio de Desarrollo Social de la Nación, continuando a cargo de dicha dependencia Claudio Morresi, quien se desempeñaba desde 2004 en el puesto ${ }^{8}$. Haciendo una evocación sobre esta incorporación la Ministra de la mencionada cartera, Alicia Kirchner (2008, p.7), entendía este cambio "[...] con visión de futuro y con gran esperanza [...]" dado "[... ] el reconocimiento y valor potencial e instrumental que le reconocemos a la actividad física, el deporte y la recreación para el desarrollo humano". En el "Plan estratégico del deporte argentino 2008-2012" se manifestó que el desarrollo del deporte es una responsabilidad del Estado, pues concibe que existe un "Derecho al deporte y a la Actividad Física" que es de "todos y todas". Con el plan se buscó producir una consolidación de una "[...] cultura de la actividad física y el deporte, que aprende con otros y de otros procurando un fin: la inclusión, integración, equidad y búsqueda de la calidad como requisito para el desarrollo nacional" (MINISTERIO DE DESARROLLO SOCIAL, 2008 a, p.3),. Al exponer el marco teórico del "Plan estratégico del deporte argentino 2008-2012" también se encuentran menciones a: la Ley del Deporte № 20.655, el carácter polisémico del término, dejando en claro en dicho apartado que: "El deporte no tienen valores en sí mismo, sino que se construyen por juicios subjetivos que emiten las personas que lo planifican, lo practican, lo conducen o sobre la base de los efectos que éstos creen obtener" (MINISTERIO DE DESARROLLO SOCIAL, 2008a, p. 10). Con este punto de partida, sobre las cuales volveremos, se conceptualizó al deporte: "[...] como una de las grandes conductas totales del hombre institucionalizadas culturalmente. Presenta un carácter eminentemente social, que se apoya en el juego, con importante contenido físico, que requiere competición y logro de una cierta performance" (MINISTERIO DE DESARROLLO SOCIAL, 2008a, p. 11).

Dentro de esta caracterización incorporó la Secretaría de Deportes de la Nación una clasificación en:

- Deporte social "es la práctica de Actividad Física y Deportivas orientadas a la población en su conjunto, sin discriminación de edad, sexo, condición física, social, cultural o étnica, [...] generadora de situaciones de inclusión..." [El destacado es mío] (MINISTERIO DE DESARROLLO SOCIAL, 2008a, p. 11);

- Desarrollo deportivo: "... Es un proceso en el que los deportistas pueden incluirse, participar y continuar con posibilidades de éxito el tránsito hacia el camino al rendimiento deportivo" (MINISTERIO DE DESARROLLO SOCIAL, 2008a, p. 11); y por último

- Deporte de Representación Nacional: "[...] actividad deportiva organizada, correspondiente al campo del Deporte Federado y de seleccionados nacionales [...]" (MINISTERIO DE DESARROLLO SOCIAL, 2008a, p. 11).

Cada una de estas clasificaciones, cuenta con un plan nacional, en donde se incorporan programas y proyectos, en nuestro caso es de interés ahondar en el "Plan Nacional de Deporte Social" (MINISTERIO DE DESARROLLO SOCIAL, 2008, p. 11). 
En el plan de deporte social, estas prácticas son incorporadas como una oportunidad para la formación integral de toda la población, para mejorar la salud, para generar fuentes de trabajo y como "una herramienta legítima para reducir los riesgos y amenazas sociales de nuestro tiempo, en particular los efectos de la pobreza" (MINISTERIO DE DESARROLLO SOCIAL, 2008, p. 2); y, al mismo tiempo, para fundamentar esta concepción se hace mención a la Ley 26.061 de Protección Integral de los Derechos de las Niñas, Niños y Adolescentes?. También, se plantea que las prácticas deportivas se convirtieron "[...] en auténticos generadores de redes sociales que contribuyen a garantizar el desarrollo humano y la cohesión social de la ciudadanía. Son un instrumento relevante de socialización, colaborando en la reconstrucción del tejido social y propiciando la organización comunitaria" (MINISTERIO DE DESARROLLO SOCIAL, 2008, p 3).

Esta área tiene como principal iniciativa al "Programa social y deportivo Juegos Nacionales Evita". En el "Plan Nacional de Deporte Social 2008-2012" al inscribir este programa, al igual que cuando lo hizo Galmarini, se expone aquí una referencia constante a los iniciados en el año 1949 diseñados por Ramón Carrillo, enfatizando el rol de Eva Duarte de Perón. En su decreto de creación 1491/2006 como en la Ley 26.462, de diciembre de 2008, vuelve a aparecer dichas referencias. En la Ley 26.462 se incluye a los "Juegos Nacionales Evita" como competencia anual, teniendo como objetivos la contribución a la formación integral del hombre; la utilización del deporte como factor de la salud física y moral de la población; y la promoción de una conciencia nacional de los valores de la educación física y del deporte. Al realizar la difusión anual de los torneos se recurre en los distintos años a distintos atletas olímpicos, quienes realizan evocaciones a sus trayectorias personales, sus logros, la adquisición de valores y las posibilidades que brinda el deporte ${ }^{10}$. Es decir, se reiteran, al igual que en los primeros años de la década de los ' 90 , las referencias a los atletas de alto rendimiento como máximos referentes de los valores y modalidad de sociabilidad que se pregona con el deporte. Siendo también recurrente la referencia al deporte como espacio de aprendizaje de determinadas formas de sociabilidad y valores que luego se podrían transferir a otras esferas sociales. Es destacable el proceso de naturalización que han sufrido los valores con los cuales son asociadas las prácticas deportivas, dado que éstos no son problematizados, salvo el comentario que se realiza en el marco teórico mencionado precedentemente, que es contrastado al momento de justificar dicho programa, planteando que: "Fueron pensados más allá de su valor deportivo intrínseco, como una herramienta de integración y promoción social" (MINISTERIO DE DESARROLLO SOCIAL, 2008b, p. 7) (El destacado es mío).

La categoría deporte social, en este caso además de las referencias compartidas a los "Campeonatos Evita" y a la Ley del deporte sancionada en 1974 durante la presidencia de Juan Domingo Perón, es actualizada por el nuevo marco legal en torno a la ampliación de derechos, comprendiéndose no solamente a partir de las distinciones con el alto rendimiento deportivo, sino que deben adquirir rasgos singulares en su implementación al plantear la accesibilidad de toda la población por concebirse como un derecho, sin "discriminación de edad, sexo, condición física, social, cultural o étnica" posibilitando a partir de su práctica la inclusión.

\footnotetext{
9 Se hace mención al artículo 20 que destaca que: "Derecho al deporte y juego recreativo. Los organismos del Estado con la activa participación de la sociedad, deben establecer programas que garanticen el derecho de todas las niñas, niños y adolescentes a la recreación, esparcimiento, juegos recreativos y deportes, debiendo asegurar programas específicos para aquellos con capacidades especiales.

10 En el video "La historia de los juegos nacionales Evita y los valores del deporte" realizado y disponible en la página web de la Secretaría de Deportes de la Nación se puede observar lo antes planteado, a partir de los relatos de tres medallistas olímpicos y paraolímpico, como son los casos de Nadia Báez, Nadadora Paraolímpica; Juan Curuchet, Ciclista Olímpico; Sebastián Crismanich,Taekwondista Olímpico. Video disponible en: https://www.youtube.com/watch?v=7mGfrccoJ o. Acceso en: 4 abr. 2015.
} 


\section{A MODO DE CIERRE: DEPORTE SOCIAL RUPTURAS Y CONTINUIDADES}

En este breve recorrido sobre la categoría deporte social en dos períodos significativos de la política argentina, auto inscriptos dentro del peronismo, se puede observar un fuerte proceso de asociación de las prácticas deportivas: con la formación de un determinado hombre, y con las políticas y lineamientos implementados durante los gobiernos de Juan Domingo Perón. Esto se percata en la referencia tanto a las palabas del ex mandatario, a los "Campeonatos Evita" que son identificados con dicho gobierno y con la "Ley del Deporte № 20.655. Al mismo tiempo se advierte un proceso de contribución de las prácticas deportivas para combatir distintas problemáticas sociales. Ambas asociaciones se encuentran sustentadas en el reconocimiento de una serie de valores tales como: solidaridad, vínculos sociales, espíritu de grupo, la valoración del esfuerzo y la constancia; que son concebidos como intrínsecos a esta práctica, por lo que en muchos casos no se encuentran problematizados, siendo los deportistas de alto rendimiento los representantes en su máxima expresión de la posesión de esos valores. La concepción sobre el deporte que presentan, lo entiende como una esfera de la vida social generadora de determinados valores, formas de socialización que se adquieren al realizar la práctica deportiva y se busca la reproducción a otras esferas de la vida social.

Al desarrollar en estos períodos la categoría deporte social vimos cómo se encuentra en un constante proceso de resignificación, siendo informada por los lineamientos políticos y marcos institucionales en la cual se inscribe en cada momento. Por un lado, fue asociada durante la década del '90 a las políticas en materia deportiva que se realizaba por fuera al alto rendimiento con el objeto, como se planeó más arriba, de buscar que "cada individuo" participe del deporte y de esa forma se incorpore al "equipo" donde "[...] cuidar el cuerpo y estar juntos constituye la meta. [...] nos referimos a fenómenos generados desde la propia comunidad que expresan la necesidad de integración social, de pertenencia a un grupo, de reconocimiento de una identidad cultural" (GALMARINI, 1992, p. 82) (El destacado es mío). El deporte social -a partir del traspaso al Ministerio de Desarrollo Social de la Nación- toma sentidos del marco internacional que reconoce a dichas prácticas como un derecho para el desarrollo humano; al tiempo que su incorporación está permanentemente asociada a la problemática de la inclusión social, punto nodal del discurso político en materia social de la mencionada cartera desde el 2007 hasta la finalización del período estudiado.

También se advierte que la mudanza en los sentidos del deporte socia/ produce cambios en las consideraciones sobre la "competencia" dentro del mismo. Como en la gestión de Galmarini se incorpora al "deporte federado" dentro de esta categoría, a partir del 2007 aunque se siguen realizando prácticas competitivas, como los Torneos Evitas, se orienta a la búsqueda de la "integración". Por ello, la ministra de Desarrollo Social Alicia Kirchner en un mensaje a los finalistas afirmaba que a "[...] los juegos no lo están viendo como una competencia, sino como una integración para la amistad". ${ }^{11 .}$

Es decir, que los lineamientos que singularizan a cada momento de la política argentina asignan algunos rasgos al deporte y al deporte social en particular, al mismo tiempo que se observan características que lo siguen inscribiendo dentro del peronismo y en la discusión del campo deportivo.

11 Extracto de las palabras de la Ministra de Desarrollo Social de la Nación Alicia Kirchner expresadas en la apertura de las finales nacionales de los "Juegos Evita" del año 2012. Disponble en: <http://www.youtube.com/watch?feature=player_embedded\&v=Z8QpML0jHXg>. Acceso en: 15 dic. 2012 
Como pudimos observar en el desarrollo, la categoría deporte social adquiere en cada una de las gestiones trabajadas significados particulares, los cuales no pueden ser circunscriptos a los lineamientos políticos e ideológicos que organizan cada uno de estos momentos. Es decir, en cada uno de los períodos este término fue apropiado, resignificado, a partir de representaciones sociales sobre el deporte provenientes de diferentes espacios sociales, al mismo tiempo que hay elementos que resisten.

\section{REFERENCIAS}

AISENSTEIN, Ángela. Deporte y Escuela ¿Separados al Nacer? Lecturas: Educación Física y Deporte, v. 3, n.11, 1998. Disponible en: <http://www.efdeportes.com/efd11a/angela.htm>. Acceso en: 10 feb. 2016.

AISENSTEIN, Ángela. La educación física en el currículo moderno o la historia de la conformación de una matriz disciplinar (argentina, 1880-1960). In: ROZENGARDT, Rodolfo (coord.). Apuntes de historia para profesores de Educación Física. Buenos Aires: Miño y Dávila, 2006. p. 69-84.

AISENSTEIN, Ángela. La Educación Física en el nuevo contexto educativo: en busca del eslabón perdido. Lecturas: Educación Física y Deporte, v.1, n.2, 1996.Disponible en: <http://www. efdeportes.com/efd2/22ang.htm>. Acceso en: 10 feb. 2016.

ALABARCES, Pablo. Los estudios sobre deporte y sociedad: objetos, miradas, agendas. In: ALABARCES, Pablo (Coord.). Peligro de gol: Estudios sobre deporte y sociedad en América Latina. Buenos Aires: CLACSO, 2000. p.11-32.

ARCHETTI, Eduardo. El potrero, la pista y el ring: las patrias del deporte argentino. Buenos Aires: Fondo de Cultura Económica, 2001.

ARCHETTI, Eduardo. Masculinidades: fútbol, tango y polo en la Argentina. Buenos Aires: Antropofagia, 2003.

ARCHETTI, Eduardo. Prologo. In: ALABARCES, Pablo; DI GIANO, Roberto; FRYDENBERG, Julio (Comp.). Deporte y Sociedad. Buenos Aires: Eudeba, 1998. p. 9-12.

ARGENTINA. Decreto Presidencial No 1.237 de 1989. Disponible en: <http://servicios.infoleg.gob.ar/ infolegInternet/anexos/0-4999/2022/norma.htm>. Acceso en: 10 feb. 2016.

ARGENTINA. Decreto Presidencial No 1491 de 2006. Disponible en: < http://servicios.infoleg.gob.ar/ infolegInternet/anexos/120000-124999/121348/norma.htm>. Acceso en: 10 feb. 2016.

ARGENTINA. Ley de Educación Nacional N 26.206. Aprobada en 2006. Disponible en: $<$ http:// servicios.infoleg.gob.ar/infolegInternet/anexos/120000-124999/123542/norma.htm>. Acceso en: 10 feb. 2016.

ARGENTINA. Ley de los Juegos Nacionales Evita N 26.462. Aprobada en 2008. Disponible en: $<$ http://servicios.infoleg.gob.ar/infoleglnternet/anexos/145000-149999/149320/norma.htm>. Acceso en: 10 feb. 2016.

ARGENTINA. Ley de Promoción de las actividades deportivas en todo el país $\mathbf{N}^{\circ}$ 20.655. Aprobada en 1974. Disponible en: <http://servicios.infoleg.gob.ar/infoleglnternet/ anexos/25000-29999/27274/norma.htm>. Acceso en: 10 feb. 2016.

ARGENTINA. Ley de Protección integral de los derechos de las niñas, niños y adolescentes No26.061. Aprobada en 2005. Disponible en: <http://servicios.infoleg.gob.ar/infoleglnternet/ anexos/110000-114999/110778/norma.htm>. Acceso en: 10 feb. 2016. 
ARGENTINA. Ley Federal de Educación N²4.195. Aprobada en 1993 Disponible en: <http:// servicios.infoleg.gob.ar/infolegInternet/anexos/15000-19999/17009/norma.htm>. Acceso en: $11 \mathrm{dec}$. 2013.

ARGENTINA. MINISTERIO DE DESARROLLO SOCIAL DE LA NACIÓN. Plan nacional de deporte social 2008-2012. 2008 b. Disponible en: <www.deportes.gov.ar/ar/site/planestrategico/. > Acceso en: 11 dic. 2013.

ARGENTINA. MINISTERIO DE DESARROLLO SOCIAL DE LA NACIÓN. Plan estratégico de deporte Argentino 2008-2012. 2008 a Disponible en: <http://deportes.gov.ar/ar/site/ planestrategico/>.. Acceso en: 11 dic. 2013.

BOURDIEU, Pierre.¿Cómo se puede ser deportista?. In: BOURDIEU, Pierre. Sociología y Cultura. México: Editorial Grijalbo, 1990. p.193-214.

DAMATTA, Roberto. Esporte na sociedade: um ensaio sobre o futebol brasileiro. In: DAMATTA, Roberto et al. Universo do Futebol. Esporte e sociedade brasileira. Rio de Janeiro: Pinakotheke, 1982. p.19-42.

FREDERIC, Sabina. Buenos vecinos, malos políticos: Moralidad y política en el Gran Buenos Aires. Buenos Aires: Prometeo Libros, 2004.

FRYDENBERG, Julio.Historia social del fútbol del amateurismo a la profesionalización. Buenos Aires: Siglo Veintiuno, 2011.

GALMARINI, Fernando. Deporte, política y cambio. Buenos Aires: Corregidor, 1992.

GEERTZ, Clifford. La descripción de densa: hacia una teoría interpretativa de la cultura. In: La interpretación de las culturas. Barcelona:Gedisa, 2003. p.19-40.

KIRCHNER, Alicia. Prólogo. In: MINISTERIO DE DESARROLLO SOCIAL. Deporte Argentino de Representación Nacional 2003-2008. Buenos Aires: Ministerio de Desarrollo Social, 2008. p.7-8.

ROSATO, Ana; BALBI, Fernando. Representaciones sociales y procesos políticos: Estudios desde la antropología social. Buenos Aires: Antropofagia, 2003.

SCHARAGRODSKY, Pablo. Juntos pero no revueltos: la Educación Física mixta en clase de género. In: AISENSTEIN, Ángela; SCHARAGRODSKY, Pablo. Tras las huellas de la Educación Física Escolar Argentina. Cuerpo, Género y Pedagogía. 1880-1950. Buenos Aires: Prometeo Libros, 2006. p.295-309.

SCHARAGRODSKY, Pablo. La constitución de la educación física escolar en la Argentina. Tensiones, conflictos y disputas con la matriz militar en las primeras décadas del siglo XX. In: SCHARAGRODSKY, Pablo (Comp.). La invención del "homo gymnasticus": Fragmentos históricos sobre la educación de los cuerpos en movimiento en Occidente. Buenos Aires:Prometeo Libros, 2011. p.441-475.

SCHARAGRODSKY, Pablo. La educación Física escolar argentina (1940-1990): de la fraternidad a la complementariedad. Anthopologica, v.22, n. 22, p. 63-92, 2004.

Apoio:

COMISIÓN DE INVESTIGACIONES CIENTIFICAS de la Provincia de Buenos Aires-UNIVERSIDAD NACIONAL DE QUILMES/UNIVERSIDAD NACIONAL DE LA PLATA. 